\title{
ANALYSIS AND SYNTHESIS FOR THE POINT AND UNITARY SPECTRA. I
}

\author{
BY N. K. NIKOL'SKIĬ
}

Communicated by Jacob Feldman, June 4, 1974

By 'spectral analysis and synthesis' we mean the following: Let $T$ be a linear operator acting in a Banach space $X$ and having a set of eigenvectors and root vectors whose (closed linear) span is $X$. Then we ask:

(A) ("analysis") Does every subspace of $X$ invariant under $T$ contain an eigenvector of $T$ ?

(B) ("synthesis") Is every such subspace spanned by the eigenvectors and root vectors lying in it?

In a long paper to appear [5], the author studies problems of spectral analysis and synthesis in connection with weighted polynomial approximation, harmonic analysis, spectral multiplicity, and unicellularity of operators. The present note and its sequel are an announcement without proofs of some of the results of three sections of [5], ${ }^{1}$ published earlier in part in Soviet publications (some obscure). Two extreme cases are treated: that in which the spectrum $\sigma(T)$ has only one point, or only one point of accumulation; and that in which $\sigma(T)$ lies in the unit circle $\Gamma=\partial \mathrm{D}=\left\{\zeta \in \mathbf{C}^{\mathbf{1}}:|\zeta|=1\right\}$, and $T$ is in some sense close to being unitary.

The results show spectral analysis (and, a fortiori, synthesis) to depend upon a certain sort of analytic "regularity" of either the resolvent of $T$ or its powers $T^{n}(n \geqslant 0)$, some sort of "smoothness" of the operator; without such restrictions, operators "in general position" will fail to have even a spanning set of eigenvectors and root vectors.

Here by definition the 'root vectors' of $T$ are those in 47A20.

AMS (MOS) subject classifications (1970). Primary 47A10; Secondary 47Bxx,

Key words and phrases. Spectral synthesis, weighted shift.

1 Copies of the author's fuller Russian summary may be obtained from Chandler Davis, Department of Mathematics, University of Toronto, Toronto M5S 1 A1, Canada. The author is grateful to Chandler Davis for the present translation and editing of his summary. 
$\bigcup_{n \geqslant 1} \operatorname{Ker}\left((T-\lambda)^{n}\right)$. We will call an operator $T$ on $X$ 'complete' in case its root vectors have closed linear span $X$ ('are complete in' $X$ ).

1. Complete extensions of compact operators. It was shown by A. S. Markus [4] that many known and some new criteria for an operator to be complete (cf. [1]) are hereditary with respect to passage to an invariant subspace, so that they actually guarantee that the operator admits spectral synthesis. On the other hand, H. L. Hamburger [3] had shown by an example that a restriction of a complete compact operator may be a 'Volterra operator', i.e., may be without eigenvectors. Our first theorem says that the phenomenon noted by Hamburger is not exceptional, that every compact operator can occur as such a restriction.

THEOREM 1. Let $T$ be a compact operator on the separable Banach space $X$. Then there exist a space $Y$ and an operator $A$ on $Y$ such that (1) $Y=X \oplus H$ with $H$ a Hilbert space; (2) $A X \subset X, A \mid X=T$; (3) $A$ is compact and complete.

The proof relies on the following "uniqueness theorem", which may have independent interest.

Lemma. Let $\lambda$ be an increasing positive function on $[0,+\infty)$ with $\lim _{r \rightarrow \infty} \lambda(r)=+\infty$. Let $A_{\infty}\left(\lambda, \mathbf{C}^{1}\right)$ denote the set of all entire functions $f$ for which $|f(\zeta)|=O(\lambda(|\zeta|))$ as $|\zeta| \rightarrow \infty$. Then there exist a nonnegative sequence $\left\{c_{n}\right\}_{n \geqslant 1}$ and a complex sequence $\left\{\zeta_{n}\right\}_{n \geqslant 1}$, with $\Sigma_{n \geqslant 1} c_{n}<\infty$ and $\lim _{n \rightarrow \infty}\left|\zeta_{n}\right|=+\infty$, such that any $f \in A_{\infty}\left(\lambda, \mathbf{C}^{1}\right)$ satisfying $\Sigma_{n \geqslant 1} c_{n}\left|f\left(\zeta_{n}\right)\right|^{2}<+\infty$ must be identically zero.

Note also that if it is given that $\|T\|<1$ or that $\sigma(T) \subset D$, then the same condition can be required of the extension of $T$ constructed in Theorem 1.

2. Unicellularity and algebras related to the shift. In light of Theorem 1, we must turn to special classes of operators. For the operators treated in this section, Question (B) comes down to the question of unicellularity; following M. S. Brodskii, one calls an operator 'unicellular' if its invariant subspaces form a chain [2].

In this section we consider weighted shifts $T=S \Lambda$ with $T e_{n}=\lambda_{n} e_{n+1}$ $(n \geqslant 0)$; here $\left\{e_{n}\right\}_{n \geqslant 0}$ is the natural basis of the sequence space $l^{p}$, and $\left\{\lambda_{n}\right\}_{n \geq 0}$ is a fixed sequence of multipliers. The operator $T^{*}$ is complete, 
its root vectors $e_{n}(n \geqslant 0)$ being complete in the appropriate space. The possibility of spectral synthesis is equivalent in this situation to unicellularity of $T^{*}$ (or, what is the same, of $T$ ), which in turn is equivalent to the condition that the discrete chain $L\left(e_{k}: 0 \leqslant k<n\right)(n=0,1, \cdots)$ should comprise all $T^{*}$-invariant subspaces.

It follows from Theorem 2 below that the property of unicellularity (synthesis) of a weighted shift depends on rather delicate "regularity" of behavior of the $\left|\lambda_{n}\right|$ (it is sufficient, for instance, that they be monotone decreasing on arithmetic progressions), and is lost if this "regularity" is slightly disturbed (for instance, monotonicity of the $\left|\lambda_{n}\right|$ on subsequences of the natural numbers sparser than the arithmetic progressions does not suffice for unicellularity). There are a number of concrete cases which can be expressed as criteria for completeness of systems of analytic functions.

In proving sufficiency of tests for unicellularity of operators $T=S \Lambda$, it is convenient to replace this condition (equivalent to completeness of the system $T^{n} x(n \geqslant 0)$ in the space $\left.l_{k}^{p}=\left\{x \in l^{p}: x_{i}=0(0 \leqslant i \leqslant k)\right\}\right)$ by the condition that $T$ be 'basic' (that the system of $T^{n} x(n \geqslant 0)$ gives a basis in $l_{k}^{p}$ equivalent to within normalization and linear isomorphism to the natural basis $\left.\left\{e_{n}: n>k\right\}\right)$. Under the condition $r(T)=\lim _{n \rightarrow \infty}\left|\lambda_{0} \cdots \lambda_{n-1}\right|^{1 / n}$ $=0$, which is necessary for unicellularity, every basic operator is unicellular.

THEOREM 2. Let $T=S \Lambda$ be a weighted shift on $l^{p}, 1 \leqslant p \leqslant \infty$; assume $\lambda_{n} \neq 0(n \geqslant 0)$, and $r(T)=0$. Then the following are equivalent: (1) $T$ is basic; (2) for every $j \geqslant 0$, the space $l^{p}\left(w_{n+j}\right)_{n \geqslant 0}$, where $w_{n}$ denotes $\lambda_{0} \cdots \lambda_{n-1}$, is a Banach algebra under convolution.

To obtain the consequences stated two paragraphs earlier, one applies the following easily verified fact: for (2) in the theorem, it is sufficient (also necessary when $p=1, \infty)$ that

$$
\sup _{m \geqslant 0}\left(\sum_{k=0}^{m}\left|\frac{w_{m+j}}{w_{k+j} w_{m-k+j}}\right|^{p^{\prime}}\right)^{1 / p^{\prime}}<\infty \quad(j \geqslant 0),
$$

where $1 / p^{\prime}+1 / p=1$.

\section{REFERENCES}

1. I. C. Gohberg and M. G. Krein, Introduction to the theory of linear nonselfadjoint operators in Hilbert space, "Nauka", Moscow, 1965; English transl., Transl. Math. Monographs, vol. 18, Amer. Math. Soc., Providence, R. I., 1969. MR 36 \#3137; 39 \#7447. 2. Theory and applications of Volterra operators in Hilbert space, 
"Nauka", Moscow, 1967; English transl., Transl. Math. Monographs, vol. 24, Amer. Math. Soc., Providence, R. I., 1970. MR 36 \#2007; 41 \#9041.

3. H. L. Hamburger, Über der Zerlegung des Hilbertschen Raumes durch vollstetige lineare Transformationen, Math. Nachr. 4 (1951), 56-69. MR 12, 718.

4. A. S. Markus, A spectral synthesis problem for operators with point spectrum, Izv. Akad. Nauk SSSR Ser. Mat. 34 (1970), 662-688 = Math. USSR 4 (1970), 670696. MR 41 \#9030.

5. N. K. Nikol'skif, Selected topics in weighted approximation and spectral analysis, Trudy Mat. Inst. Steklov. 120 (1974). (Russian)

LENINGRAD BRANCH OF THE STEKLOV INSTITUTE (LOMI), FONTANKA 25, LENINGRAD D-11, USSR 